\title{
IMPLICAÇÕES BIOPSICOSSOCIAIS DA DOR ONCOLÓGICA
}

\author{
BIOPSYCHOSOCIAL IMPLICATIONS OF ONCOLOGICAL PAIN
}

\author{
Rauana dos Santos Faustino, Jackeline Kérollen Duarte de Sales, Dailon de Araujo Alves, \\ George Pimentel Fernandes
}

Universidade Regional do Cariri - URCA

\begin{abstract}
Objective: To understand, through the available evidence in the literature, as biopsychosocial implications of cancer pain. Method: This is an integrative literature review with a qualitative approach. For a research study, we used the descriptors "pain" and "neoplasia", interspersed by the Boolean operator AND, published between 2012 and 2016, in the Scielo virtual library. To include, opt for studies available in full and in the Portuguese language. Results: 12 studies were selected that addressed the theme in question. Four thematic categories related to "Feelings" and patient experiences submitted to "Procedures", "Family of patients" and "Professionals" that accompany close treatment, as well as Nursing are essentially eligible. Conclusions: The cancer patient breaks down a series of phenomena, not only presents the fear of death, but still makes a confrontation with painful, familial changes, monitoring of his emotional state and coping with a pain.
\end{abstract}

\section{Resumo}

Objetivo: compreender, por meio das evidências disponíveis na literatura, as implicações biopsicossociais da dor oncológica. Método: trata-se de uma revisão integrativa da literatura, de abordagem qualitativa. Para a busca dos estudos, foram utilizados os descritores "dor" $e$ "neoplasia", intercalados pelo operador booleano AND, publicados entre os anos de 2012 à 2016, na biblioteca virtual do Scielo. Para inclusão, optou-se por estudos disponíveis na íntegra e no idioma português. Resultados: foram selecionados 12 estudos que tratavam da temática em questão. Elegeram-se quatro categorias temáticas que discutiram os aspectos relacionados aos "Sentimentos" e vivências do paciente que é submetido aos "Procedimentos", a "Família do paciente" e "os profissionais" que acompanham de perto o tratamento, bem como a enfermagem em sua essência de cuidar. Conclusões: o paciente oncológico desmembra uma série de fenômenos, não bastando apenas o medo da morte, mas ainda todo um confronto com tratamentos dolorosos, aceitação familiar, monitoramento do seu estado emocional e o lidar com a dor.
Palavras chave: Dor; Neoplasia; Família; Enfermagem 
Introdução

Câncer é um conjunto de doenças que tem em comum o crescimento desordenado de células que invadem os tecidos e órgãos, podendo espalhar-se para outras regiões do corpo (metástase). Essas células tendem a ser extremamente agressivas e incontroláveis, pois tem o seu desenvolvimento acelerado, são invasivas, levando a destruição dos tecidos adjacentes, podendo desenvolver metástase regional e a distância; e em sua maioria, resistentes ao tratamento podendo levar o indivíduo a morte ${ }^{1,2}$. Quanto à isto, tem sido evidente o aumento gradual dos índices de diagnóstico de câncer em todo o mundo e estima-se a ocorrência de cerca de 600 mil casos novos de câncer no Brasil para cada ano de 2018/20193.

Com relação ao perfil do câncer, destaca-se a presença da dor como sendo um sintoma comum frente a este contexto. Sendo habitual o surgimento desta, desde o momento do diagnóstico (psicológica), que se segue após cirurgias de remoção de tumor (biológica), durante as sessões de radioterapia e quimioterapia, até a sua disseminação, expressando-se pelo crescimento do tumor e pressão nos órgãos, condicionados pelo abcesso ${ }^{4}$. O controle dessa dor é um processo complexo, que requer a avaliação dos componentes de ordem física, social, espiritual, econômica, emocional e cultural ${ }^{5}$.

A presença dos familiares e profissionais da saúde compõe um ambiente social ao qual o sujeito com neoplasia está inserido. Esses sujeitos (profissionais e familiares) que convivem com o paciente, também desenvolvem sentimentos relacionados à dor do outro. Dentro do contexto familiar, é estabelecido no desenvolvimento de cada indivíduo normas e valores, onde para cada membro se instituem papeis em função do desenvolvimento da vida social $^{6}$. Nisso, aquele que exerce o papel de responsável aos cuidados do enfermo, o familiar cuidador, torna-se suporte para a dor oriunda do câncer.

Destarte, mesmo que recorrente em pacientes oncológicos, a expressão da dor varia não somente de um indivíduo para o outro, mas também de acordo com suas diferentes culturas, consistindo, portanto, em algo conclusivamente subjetivo, o que acaba por dificultar o entendimento da dor de origem oncológica em sua totalidade 7 . Nisso, os mecanismos de enfrentamento tanto pelos profissionais e familiares que convivem com a dor do paciente, os procedimentos que são dispostos a minimizar a dor e os sentimentos que os próprios pacientes vivem, constituem um contexto amplo que envolve o meio biológico, psicológico e social.

Deste modo, esta produção científica busca evidenciar a dor de origem oncológica com ênfase em aspectos emocionais e de convívio social, contribuindo para as indagações pertinentes ao assunto, à medida que se busca relatar as experiências da dor e o câncer vivenciados pelos pacientes, familiares e profissionais da saúde. Para isto, objetivou-se compreender por meio das evidências disponíveis na literatura, as implicações biopsicossociais da dor oncológica.

\section{Metodologia}

Trata-se de uma revisão integrativa da literatura, de abordagem qualitativa, realizada entre os meses de março e maio de 2016. Possui caráter exploratório, priorizando a análise textual de diferentes autores que dividem ideias sobre casos clínicos de dor oncológica, fenômenos ocorrentes na presença de familiares, profissionais e as diferentes reações de pacientes nessa condição.

O estudo evidencia principalmente a relação entre "dor" AND "neoplasia", descritores usados na busca que se atentou à base de dados da Scielo. Para os critérios de inclusão, priorizaram-se produções em texto completo, dispostos em língua portuguesa (a fim de identificar as publicações nacionais) e publicados nos últimos cinco anos, de 2012 à 2016. Foram excluídos os artigos repetidos, e estudos do tipo monografia e dissertação.

Na primeira etapa, foi elaborado um quadro síntese para cada categoria gerada, contendo título, objetivo e resultado, visando melhorar a visualização das ideias dos autores e os dados coletados. Diante dos achados, elencou-se as seguintes categorias: A convivência da dor oncológica no contexto familiar, Minimizando a dor oncológica, A dor oncológica no contexto dos profissionais, e Os sentimentos vivenciados pelos pacientes com dor oncológica.

$\mathrm{Na}$ segunda etapa da fase qualitativa, a análise concretiza-se pela identificação de ideias centrais, avaliação dos artigos, compilação e comparação de ideias sobre as esferas destacadas, havendo uma produção textual sobre cada categoria, contendo, inclusive, citações dos escritores selecionados. 


\section{Resultados e discussão}

Foram encontrados 126 estudos, após a aplicação dos critérios de inclusão e exclusão, permaneceram 16 artigos, foram ainda excluídos 04 estudos que após leitura dos títulos e resumos, não trataram da temática em estudo, permanecendo 12 artigos científicos, identificados no Quadro 01. A partir dos dados encontrados, elegeram-se categorias temáticas as quais serão discutidas adiante.

Quadro 1. Artigos selecionados e analisados, segundo ano de publicação, autoria e titulo.

\begin{tabular}{|c|c|c|}
\hline Ano & Autores & Título do Estudo \\
\hline 2012 & Costa AIS, Chaves MD & Dor em pacientes com câncer sob tratamento quimioterápico. \\
\hline 2012 & $\begin{array}{l}\text { Bezerra TS, Rett MT, } \\
\text { Mendonça ACR, Santos } \\
\text { DE, Prado VM, Santana } \\
\text { JM. }\end{array}$ & $\begin{array}{l}\text { Hipoestesia, dor e incapacidade no membro superior após radioterapia } \\
\text { adjuvante no tratamento para câncer de mama }\end{array}$ \\
\hline 2012 & $\begin{array}{l}\text { Marmo MCR, CARAN } \\
\text { EMM, PUTY FCB, MORAIS } \\
\text { MB. }\end{array}$ & $\begin{array}{l}\text { Avaliação do hábito intestinal em pacientes com câncer que utilizam } \\
\text { morfina para o controle da dor }\end{array}$ \\
\hline 2012 & $\begin{array}{l}\text { Minson FP, Assis FD, } \\
\text { Venatti TK, Junior JS, } \\
\text { Mateus WP, Giglio AD. }\end{array}$ & Procedimentos intervencionistas para o manejo da dor no câncer \\
\hline 2012 & $\begin{array}{l}\text { Rett MT, Mesquita PJ, } \\
\text { Mendonça ARC, Moura } \\
\text { DP, Santana JM. }\end{array}$ & $\begin{array}{l}\text { A cinesioterapia reduz a dor no membro superior de mulheres submetidas } \\
\text { à mastectomia ou quadrantectomia }\end{array}$ \\
\hline 2013 & $\begin{array}{l}\text { Barbosa Neto JO, Sousa } \\
\text { MA, Tahamtani SMM, } \\
\text { Ashmawi HA. }\end{array}$ & $\begin{array}{l}\text { Bloqueio neurolítico subaracnoideo em paciente com dor oncológica } \\
\text { refratária }\end{array}$ \\
\hline 2013 & $\begin{array}{l}\text { Carvalho MWA, Nobrega } \\
\text { MML, Garcia TR. }\end{array}$ & $\begin{array}{l}\text { Processo e resultados do desenvolvimento de um Catálogo } \mathrm{CIPE}^{\circledR} \text { para dor } \\
\text { oncológica }\end{array}$ \\
\hline 2013 & $\begin{array}{l}\text { Macedo ACPA, Romanek } \\
\text { FVRM, Avelar MCQ. }\end{array}$ & $\begin{array}{l}\text { Gerenciamento da dor no pós-operatório de pacientes com câncer pela } \\
\text { enfermagem }\end{array}$ \\
\hline 2013 & $\begin{array}{l}\text { Motta MGC, Diefenbach } \\
\text { GDF. }\end{array}$ & $\begin{array}{l}\text { Dimensão da vulnerabilidade para as famílias da criança com dor } \\
\text { oncológica em ambiente hospitalar }\end{array}$ \\
\hline 2013 & Rabelo ML, Borella MLL. & $\begin{array}{l}\text { Papel do farmacêutico no seguimento farmacoterapêutico para o controle } \\
\text { da dor de origem oncológica }\end{array}$ \\
\hline 2015 & $\begin{array}{l}\text { Rodrigues LA, Moraes EL, } \\
\text { Betoschi JR, Amaral CP }\end{array}$ & $\begin{array}{l}\text { Como a dor e o sofrimento do paciente oncológico afetam o médico no } \\
\text { processo de tratamento }\end{array}$ \\
\hline 2015 & $\begin{array}{l}\text { Siqueira HBOM, Santos } \\
\text { MA, Gomez RRF, Saltareli } \\
\text { S, Sousa FAEF. }\end{array}$ & $\begin{array}{l}\text { Expressão da dor na criança com câncer: uma compreensão } \\
\text { fenomenológica. }\end{array}$ \\
\hline
\end{tabular}

Fonte: Autores, 2019

\section{A convivência da dor oncológica no contexto familiar}

Nesse contexto os estudos possibilitaram uma compreensão sobre os parâmetros que envolvem os aspectos vividos pelos familiares de pacientes com dor de origem oncológica.

É vigente e inquietante o assunto da dor, literaturas trazem esse assunto, e entre elas surgem às relações dor, câncer e a postura do paciente no seu dia-a-dia. Muitos pacientes ainda acabam experimentando mudanças de status no seu emprego, nas relações sociais, na sua capacidade física e no seu papel dentro da família ${ }^{8}$. Mudanças que vão desde a perda do papel 'provedor da família' até a 'dependência no autocuidado'.
Nota-se a existência de duas esferas de relação entre paciente e família que são pontos de estudo. O acometido frente sua posição familiar e o comportamento da família ao ter que lidar com a nova realidade, onde os sentimentos mais delicados podem surgir. Diversas e imprevisíveis são as reações das famílias frente à doença e à hospitalização de seus filhos e parentes, dentre estes: medo, insegurança, culpa, ansiedade pelo diagnóstico e cura ${ }^{9}$.

$A$ realidade daqueles que convivem com o paciente se transforma e isto pode variar, a depender do discernimento de cada pessoa, em que devem processar as diversas informações advindas de todos os lados, maneiras, pessoas, profissionais da saúde e, ainda, continuar 
eficiente, na tarefa de estar junto e cuidar de seu doente com dor ${ }^{10}$.

Já as crianças, quando são alvos dessa enfermidade, retratam uma percepção diferenciada com relação a um adulto e o comportamento do familiar frente às situações onde ser familiar de criança com dor oncológica é estar vulnerável duplamente ${ }^{10}$. Nisso o câncer infantil repercute também no sistema familiar, desencadeando por vezes nos outros membros familiares, o medo da dor, do sofrimento, da mutilação e da iminência da finitude ${ }^{9}$.

São situações, delicadas e dimensionadas à esfera emotiva, no indivíduo com dor, como protagonista que sofre, é nesse contexto que surge a família também como fonte de cuidados. A família revela-se essencial no tratamento da dor em pacientes com câncer. E este tratamento que deve ser elaborado por uma equipe multiprofissional, equipes médica e de enfermagem, psicólogos, assistentes sociais, religiosos e outros, bem como o próprio paciente e sua família participam do processo dinâmico ${ }^{11}$.

\section{Minimizando a dor oncológica}

Essa categoria trata dos relatos sobre os procedimentos a serem realizados com o próprio paciente que sofre consequências dolorosas em decorrência do câncer.

São citados na literatura alguns procedimentos na intervenção da dor em pacientes oncológicos, intercalando de cirurgias a fármacos, fisioterapia e diálogo entre profissionais e pacientes. Mencionam-se vários procedimentos, além da cirurgia, tratamentos complementares, como a quimioterapia (QT), a radioterapia (RT) e/ou a hormonioterapia podem ser empregados ${ }^{12}$. A fisioterapia destaca-se como uma importante ferramenta na reabilitação após a cirurgia, principalmente no tratamento do câncer de mama. A prática da cinesioterapia após intervenções cirúrgicas, além de outras intervenções em fisioterapia, tem fundamental importância na prevenção e tratamento da dor ${ }^{11}$.

As literaturas questionam sobre a real eficácia dos tratamentos, postulando entre elas, taxas de seu funcionamento. Alguns procedimentos conseguem tratar efetivamente $80 \%$ à $90 \%$ dos pacientes com terapia farmacológica. No entanto, $10 \%$ à $20 \%$ dos pacientes com câncer não respondem adequadamente aos fármacos e se beneficiam das terapias intervencionistas de dor ${ }^{14}$. Mesmo com a utilização dos fármacos e terapias complementares, nem sempre há sucesso na supressão da dor ${ }^{8}$.
A dor decorrente do câncer é, em geral, passível de tratamento, mas uma gestão correta exige um conhecimento da fisiopatologia da dor, da farmacologia dos analgésicos e do manejo das questões psicossociais ${ }^{15}$.

Esse mesmo pensamento é dividido entre os vários autores que relacionam a eficácia do tratamento com as relações ambiente, profissional, família e indivíduo. O controle da dor é um processo complexo que requer a avaliação dos componentes de ordem física, social, espiritual, econômica, emocional e cultural ${ }^{5}$.

A farmacologia e a fisioterapia são citadas de forma frequente entre as intervenções no tratamento da dor, ao seu tempo e de forma segura. O uso de terapia intervencionista em momento apropriado, pode permitir uma drástica redução do consumo de opioides e reduzir a morbidade causada pela dor para este grupo de pacientes ${ }^{14}$.

Em casos específicos, como o tratamento com crianças, relata-se uma atenção diferenciada visando o psicossocial da criança. A importância dessa atenção, coloca que o alívio da dor e a atenção dada aos aspectos sociais da doença devem estar inseridos na atenção integral que a criança em processo de adoecimento deve receber $^{10}$. O pesquisador e o profissional de saúde necessitam desenvolver uma atitude empática, sensível e apropriada à capacidade cognitiva e emocional da criança para, assim, compreender o ser "sofre-dor" em sua totalidade.

Essa forma de pensar também está inserida frente a outros pesquisadores e que também levam esse raciocínio a outros quadros de dor, como adultos, por exemplo, e como consequências, lidar com as várias reações oriundas desses pacientes. No trabalho de cuidar de pessoas, os profissionais, ao formarem vínculos afetivos com seus pacientes, precisam encontrar formas de lidar com o impasse de ter um distanciamento afetivo que lhes permita cumprir a sua função e, ao mesmo tempo, se proteger ${ }^{11}$.

A Organização Mundial da Saúde (OMS) também faz referências sobre o assunto e caracteriza um dos processos corretos para intervir na dor. Através do manejo de uma equipe multidisciplinar, a qual deve seguir o protocolo "Guia para o tratamento da Dor no Câncer" proposto pela (OMS), e utilizar fármacos por via oral de acordo com a Escala Analgésica ${ }^{16}$.

Os artigos têm citado experiência de pacientes com dor oncológica, onde tem-se relatado a presença da espiritualidade como recurso que desencadeia efeitos positivos para o 
alívio da dor ${ }^{9}$.

\section{A dor oncológica no contexto dos profissionais}

Em relação aos profissionais da saúde de forma geral, essa categoria aborda a perspectiva, enquadrando relatos, questionamentos frente ao profissional e o paciente oncológico em tratamento contra a dor.

Questionar a correta atuação do profissional, a relação no seu ambiente de trabalho, é assunto para várias literaturas, as quais discutem capacitações e discernimento para se sobressair nas mais variadas situações. Os profissionais capacitados no controle da dor possuem maiores possibilidades de garantir uma assistência mais humanizada ao paciente e dessa forma, torna-se de extrema importância capacitar os profissionais de saúde em relação ao seu manuseio ${ }^{17}$.

Por sua vez, o assunto humanização está direcionado a relação do profissional com o paciente, viabiliza resultados, seja no convívio ou no sucesso do tratamento, sendo um conjunto de alternativas viáveis, capacitação e humanização. Os autores estudados trazem apenas alguns aspectos que englobam o papel do profissional no tratamento de dor. A forma de atuar dos profissionais de saúde comunica-se entre si, e como lidam com a dor do paciente são aspectos influenciados por suas definições ${ }^{8}$.

O sofrimento do paciente pode trazer incômodos para os profissionais, tendo em vista a humanidade que cada um possui. Em uma das literaturas abre-se um parêntese sobre como é a relação médico e enfermo com dor. Por sua vez a Oncologia Clínica, refere-se à especialidade da Medicina envolvida no processo de diagnóstico e tratamento do câncer, prevenção da doença e reabilitação do paciente recuperado, afeta a qualidade da relação médico-paciente, podendo causar expectativas ilusórias nos pacientes e familiares, e também provocar sofrimento ao médico ${ }^{11}$.

Abre-se uma importância no papel do enfermeiro na recuperação do paciente, onde se destina a esse profissional o trabalho de atenção. Diariamente, todos os pacientes oncológicos devem ser avaliados quanto à presença e intensidade da dor, atualmente, devido à proximidade da equipe de enfermagem com o paciente, os enfermeiros são mais bem indicados ${ }^{16}$.

O manuseio, a sensibilidade e a percepção da equipe de enfermagem são essenciais para a avaliação e a assistência no tratamento adequado em relação ao paciente com dor ${ }^{17}$.
Tem sido relevante a capacitação dos enfermeiros para o cuidado do paciente ${ }^{5}$.

É notória a participação que deve ser exercida pelo profissional de enfermagem, desse modo o enfermeiro deve exercer seu papel no controle da dor, ter responsabilidade na avaliação diagnóstica, na intervenção e monitorização do tratamento por meio da comunicação com o paciente ${ }^{8}$.

Atribuições feitas à equipe de enfermagem configuram um leque de possibilidades, tendo em vista as variações de pacientes e suas personalidades, como o trabalho direto com crianças, o controle da dor na criança com câncer, bem como a minimização do sofrimento, fazem parte do ser, saber-fazer em Enfermagem ${ }^{17}$.

Os sentimentos vivenciados pelos pacientes com dor oncológica

Essa categoria busca sistematizar citações que estabeleçam um olhar emocional, acerca do indivíduo enfermo ou das pessoas ao seu redor.

O sintoma dor merece atenção por estar diretamente relacionado ao bem-estar físico e emocional, ao desempenho e realização das atividades da vida diária (AVD), bem como a qualidade de vida. Situações novas, etapas de adaptação que nem sempre são concluídas $^{13}$, o paciente começa a vivenciar um mundo novo, cheio de conflitos, regras, imposições, dúvidas, incertezas e questionamentos ${ }^{10}$.

A variação de sentimentos expressa é alarmante, sensação de desesperança, perdem o sentindo da vida, surge à ansiedade e sintomas depressivos, a perda da autoestima ${ }^{8}$. Outros autores dividem a mesma linha de estudo e relatam fielmente a postura de pacientes oncológicos e fazem uma relação da dor com a doença propriamente dita. $O$ sofrimento incalculável, redução da qualidade de vida, podem levar os pacientes a temerem mais o sintoma doloroso que o próprio câncer ${ }^{5}$. Os sentimentos como depressão, ansiedade, catastrofização e cognição podem influenciar a percepção da dor e contribuir para a intensidade da dor total ${ }^{15}$.

Uma das literaturas chama atenção sobre um relato em especial de um paciente, onde a dor se sobressaiu diante da integridade física, um paciente que já apresentava grande limitação para deambulação e que para ele o benefício do alívio da dor superava o risco de bloqueio motor permanente ${ }^{14}$.

O quadro de vulnerabilidades que alguns pacientes apresentam a doença, a incapacidade e o sofrimento são algumas das circunstâncias que 
conferem estado de vulnerabilidade e são condições que requerem o cuidado ${ }^{10}$. As crianças podem estar envolvidas nessa definição e também "expressam sentimentos de terror em relação à dor, medo da morte, graves alterações biopsicossociais, onde questões espirituais, emocionais e culturais se entrelaçam" ${ }^{\prime \prime}$.

\section{Conclusão}

Mesmo com o aumento nos casos de câncer e de pesquisas relacionadas, percebe-se que ainda há um campo vasto a ser pesquisado. $O$ paciente enquanto indivíduo desmembra uma série de contextos e fenômenos aos quais está inserido, não bastando apenas o medo da morte (sentimentos), mas ainda todo um confronto com tratamentos dolorosos (biológico), a aceitação familiar (contexto familiar) e o lidar com a dor.

A dor de origem oncológica, gera implicações biopsicossociais, com interpretações distintas entre as diversas faixas etárias, principalmente no que diz respeito a sua identificação e manejo, que se considerados em tempo hábil e oportuno podem gerar prognósticos mais resolutivos, assim como, uma adesão terapêutica mais satisfatória por parte dos pacientes.

Em suma, esse estudo possibilitou um encontro de parâmetros e evidências importantes, que oferecem uma ampla compreensão sobre a dor de origem oncológica e integra séries ocorrentes no quadro neoplásico, regras de convívio e reações de familiares, profissionais e do próprio paciente. O estudo encontrou limitação ao excluir estudos não disponíveis na integra, que restringiu a amostra final de estudos.

\section{Referências}

1. Sampaio JF, Costa MF. Métodos para o tratamento da dor em crianças com câncer. Revista discente da UNIABEU. 2017 Jul/Dez; 5(10). Disponível em: <http:// https://revista.uniabeu.edu.br/index.php/alu/art icle/view/2451/2112> Acesso em: 18 de setembro de 2019.

2. INCA, Instituto nacional do Câncer. Disponível em: < http://www2.inca.gov.br/wps/wcm/connect/co municacaoinformacao/site/home> Acesso em: 25 de Julho de 2016.

3. INCA, Instituto nacional do câncer. ABC do câncer: abordagens básicas para o controle do câncer. Rio de Janeiro. 2019. Disponível em: < https://www.inca.gov.br/sites/ufu.sti.inca.local/fi les//media/document//livro-abc-4-edicao.pdf>. Acesso em: 18 de setembro de 2019.

$4 . \quad$ Instituto Oncoguia. Dor. Disponível em : <http://www.oncoguia.org.br/conteudo/dor/208 /109/ > Acesso em: 05 de Agosto de 2016.

5. Carvalho MWA, Nobrega MML, Garcia TR. Processo e resultados do desenvolvimento de um Catálogo $\mathrm{CIPE}^{\circledR}$ para dor oncológica. Rev Esc Enferm USP, São Paulo. 2013; 47(5): 1061-8. Disponível em: <http://www.scielo.br/pdf/reeusp/v47n5/pt_008 0-6234-reeusp-47-05-1060.pdf>. Acesso em: 24 nov 2018.

6. Pareja JMD, Guerra FF, Vieira SR, Teixeira KMD. A produção do espaço e sua relação no processo de saúde - doença familiar. Saúde Soc. São Paulo. 2016; 25(1): 133-144. Disponível em: < http://ref.scielo.org/czxdbx>. Acesso em: 24 nov 2018.

7. SBED, Sociedade Brasileira Para Estudo da Dor. O que é dor? . Disponível em: < http://www.sbed.org.br/materias.php?cd_secao =76\&codant $=$ \&friurl=_-O-que-e-Dor-_ > Acesso em: 05 de Agosto de 2016.

8. Costa AIS, Chaves MD. Dor em pacientes com câncer sob tratamento quimioterápico. Rev Dor, São Paulo. 2012 jan./mar; 13(1): 45-9. Disponível em: < http://ref.scielo.org/v8dswd>. Acesso em: 24 nov 2018.

9. Siqueira HBOM, Santos MA, Gomez RRF, Saltareli S, Sousa FAEF. Expressão da dor na criança com câncer: uma compreensão fenomenológica. Estudos de Psicologia, Campinas. 2015. out./dez.; 32(4): 663-674. Disponível em: < http://ref.scielo.org/45gybr>. Acesso em: 24 nov 2018.

10. Motta MGC, Diefenbach GDF. Dimensões da vulnerabilidade para as famílias da criança com dor oncológica em ambiente hospitalar. Esc Anna Nery (impr.). 2013. jul./set.; 17(3): 482-490. Disponível em: < http://ref.scielo.org/b7rrgs>. Acesso em: 24 nov 2018.

11. Rodrigues LA, Moraes EL, Betoschi JR, Amaral CP. Como a dor e o sofrimento do paciente oncológico afetam o médico no processo de tratamento. Cuidarte Enfermagem. 2015. jan./jun.; 9(1): 58-70. Disponível em: < http://fundacaopadrealbino.org.br/facfipa/ner/p $d f /$ Revistacuidarteenfermagem\%20v.\%209\%20n. 1\%20\%20jan.\%20jun\%202015.pdf>. Acesso em: 24 nov 2018.

12. Bezerra TS, Rett MT, Mendonça ACR, Santos DE, Prado VM, Santana JM. Hipoestesia, dor e incapacidade no membro superior após radioterapia adjuvante no tratamento para 
câncer de mama. Rev Dor. São Paulo. 2012. out./dez.; 13(4): 320-6. Disponível em: <http://www.scielo.br/pdf/rdor/v13n4/03.pdf>. Acesso em: 24 nov 2018.

13. Rett MT, Mesquita PJ, Mendonça ARC, Moura DP, Santana JM. A cinesioterapia reduz a dor no membro superior de mulheres submetidas à mastectomia ou quadrantectomia. Rev Dor, São Paulo. 2012. jul./set.; 13(3): 201-7. Disponível

em: <http://www.scielo.br/pdf/rdor/v13n3/v13n3a0 2>. Acesso em: 24 nov 2018.

14. Barbosa Neto JO, Sousa MA, Tahamtani SMM, Ashmawi HA. Bloqueio neurolítico subaracnoideo em paciente com dor oncológica refratária. Rev Dor, São Paulo. 2013. jan./mar.; 14(1): 76-7. Disponível em: < http://ref.scielo.org/z75ss6>. Acesso em: 24 nov 2018.

15. Minson FP, Assis FD, Venatti TK, Junior JS, Mateus WP, Giglio AD. Procedimentos intervencionistas para o manejo da dor no câncer. Einstein, Campinas - São Paulo, v.10, n.3, p. 2925, 2012. Disponível em: < http://www.scielo.br/pdf/eins/v10n3/v10n3a06>. Acesso em: 24 nov 2018.

16. Rabelo ML, Borella MLL. Papel do farmacêutico no seguimento farmacoterapêutico para o controle da dor de origem oncológica. Rev Dor, São Paulo. 2013. jan./mar.: 14(1): 58-60. Disponível em: < http://ref.scielo.org/26kfsw>. Acesso em: 24 nov 2018.

17. Macedo ACPA, Romanek FVRM, Avelar MCQ. Gerenciamento da dor no pós-operatório de pacientes com câncer pela enfermagem. Rev Dor, São Paulo. 2013. abr./jun.; 14(2): 133-6. Disponível em: <http://ref.scielo.org/8cx3xx>. Acesso em: 24 nov 2018.

\section{Endereço para Correspondência}

Universidade Regional do Cariri - URCA

Rua Coronel Antônio Luíz, 1161 - Pimenta

Crato - CE

CEP.: 63105-010

e-mail: jackelinekerollen@hotmail.com

Recebido em 30/11/2018

Aprovado em 24/09/2019

Publicado em 13/02/2020 